\title{
$\mathrm{AD} / \mathrm{RHIC} / \mathrm{AP}-88$
}

\author{
RHIC PROJECT \\ Brookhaven National Laboratory
}

Effects of Position Errors of the Magnetic Center in Dipoles

\author{
G. Parzen
}

January 1990 


\section{Effects of Position Errors of the Magnetic Center in Dipoles \\ G. Parzen}

Because of the systematic $b_{2}$ due to magnetization or iron saturation, random position errors in the magnetic center will generate a random $b_{1}$.

The $b_{2}$ due to magnetization or saturation in the dipoles is

\begin{tabular}{|c|c|c|c|c|}
\hline$\gamma$ & 7 & 12 & 100 & \\
\hline$b_{2}^{\prime}$ & -10 & -3 & -6.5 & $\times 10^{-4}$ \\
\hline
\end{tabular}

The expected random $b_{1}$ in the dipoles is $b_{1}=8.4 \times 10^{-5} / \mathrm{cm} \mathrm{rms}$. It is hoped to reduce this $b_{1}$ by about a factor of 4 by magnet shuffling. In order to preserve this factor of 4 , the random magnetic center error should lead to a random $b_{1}$ which is smaller than $b_{1}=\frac{1}{4}\left(8.4 \times 10^{-5}\right)$ or $b_{1}=2.1 \times 10^{-5} / \mathrm{cm} \mathrm{rms}$.

A $0.5 \mathrm{~mm} \mathrm{rms} \mathrm{error} \mathrm{in} \mathrm{the} \mathrm{magnetic} \mathrm{center} \mathrm{will} \mathrm{give} \mathrm{a} \mathrm{random} b_{1}$ of $b_{1}=1.46 \times 10^{-5} / \mathrm{cm}$ rms.

Assuming a closed orbit error in the dipoles of $0.5 \mathrm{~mm} \mathrm{rms}$, then the magnetic center is off the closed orbit by

$$
\Delta x=\sqrt{\left.(0.5)^{2}+(0.5)^{2}\right)}=0.7 \mathrm{~mm} / \mathrm{rms}
$$

and

$$
b_{1}=2\left(\frac{6.5}{6.25}\right) \times 10^{-4} \times 0.07=1.46 \times 10^{-5} / \mathrm{cm} \mathrm{rms}
$$

using the $b_{2}^{\prime}=6.5$ at $\gamma=100$.

The average $b_{1}$ generated, as described above, can be corrected by horizontal positioning of the dipole during installation, as suggested by H. Hahn. A $0.5 \mathrm{~mm}$ rms error in the dipole installation may be considered as a possible tolerance. 